\title{
Symbol Synchronization Exploiting the Symmetric Property in Optical Fast OFDM
}

\author{
Jian Zhao, Selwan K. Ibrahim, Danish Rafique, Paul Gunning, and Andrew Ellis
}

\begin{abstract}
We propose a new simple method to achieve precise symbol synchronization using one start-of-frame (SOF) symbol in optical fast orthogonal frequency division multiplexing (FOFDM) with sub-channel spacing equal to half of the symbol rate per sub-carrier. The proposed method firstly identifies the SOF symbol, then exploits the evenly symmetric property of the discrete cosine transform in FOFDM, which is also valid in the presence of chromatic dispersion, to achieve precise symbol synchronization. We demonstrate its use in a $16.88 \mathrm{Gbit} / \mathrm{s}$ phase shifted keying based FOFDM system over a $124 \mathrm{~km}$ field-installed single-mode fiber link and show that this technique operates well in automatic precise symbol synchronization at an optical signal-to-noise ratio as low as $3 \mathrm{~dB}$ and after transmission.
\end{abstract}

Index Terms - Modulation, orthogonal frequency division multiplexing, wavelength division multiplexing, detection

\section{INTRODUCTION}

$\mathrm{O}$ ptical fast orthogonal frequency division multiplexing (FOFDM) [1], with sub-channel spacing reduced to half of that in conventional OFDM, is a promising multi-carrier scheme, whose advantages come to light recently in wireless [2-3]. The excellent energy concentration property of discrete cosine transform (DCT) results in enhanced robustness to residual frequency offset [2] and improved performance in channel estimation [3] etc., when compared to conventional OFDM. However, the diagonalizing property of the discrete Fourier transform (DFT) is not applicable to the DCT, such that the timing error from imprecise symbol synchronization in FOFDM cannot be compensated with one-tap equalizer after sub-channel demultiplexing. Consequently, precise timing estimation is required before demultiplexing to align the DCT window at the correct position within the sequence of the received samples. Unfortunately, very little work on symbol synchronization has been reported in either optical or wireless systems [4]. Although methods for conventional OFDM were proposed [5-8], different properties between DCT and DFT require re-examination of these methods, and urge the proposal of an effective solution specific to FOFDM. In conventional OFDM, the method proposed by Schmidl is commonly used [5].

Manuscript received Dec. 4, 2010. This work was supported by Science Foundation Ireland under grant number 06/IN/I969.

Jian Zhao, Selwan K. Ibrahim, Danish Rafique, and Andrew Ellis are with Photonic Systems Group, Tyndall National Institute, University College Cork, Lee Maltings, Prospect Row, Cork, Ireland (e-mail: jian.zhao@tyndall.ie).

Paul Gunning is with BT Innovate \& Design, Adastral Park, Suffolk IP5 3RE, United Kingdom.

Digital Object Identifier: $\mathrm{xxxx}$
It uses a start-of-frame (SOF) symbol to identify the start of the sequence. However, it cannot achieve accurate timing recovery. To overcome this problem, an improved metric based on Hermitian symmetry was proposed that yielded a sharp peak at the symbol boundary [7]. However, as will be shown in this paper, this method is not resilient to chromatic dispersion (CD). Alternatively, precise symbol synchronization can be obtained by a method using subtraction and Gaussian-windowing [8]. However, a large number of training symbols is required.

We recently proposed symbol synchronization for optical FOFDM using a maximum likelihood estimation method [4]. However, this method is still somewhat complex. In this paper, we propose a new method, which is simpler and more intuitive than that reported in [4]. Furthermore, the DCT uses only real arithmetic in contrast to the DFT in conventional OFDM, whose output is complex even when the input is real. This enables the proposed method more robust to $\mathrm{CD}$ than that in [7]. We demonstrate a FOFDM experiment using $124 \mathrm{~km}$ of field installed fiber, and show that the proposed method operates well for automatic symbol synchronization at an optical signal-tonoise ratio (OSNR) as low as $3 \mathrm{~dB}$ and in the presence of $\mathrm{CD}$.

\section{PRINCIPLE}

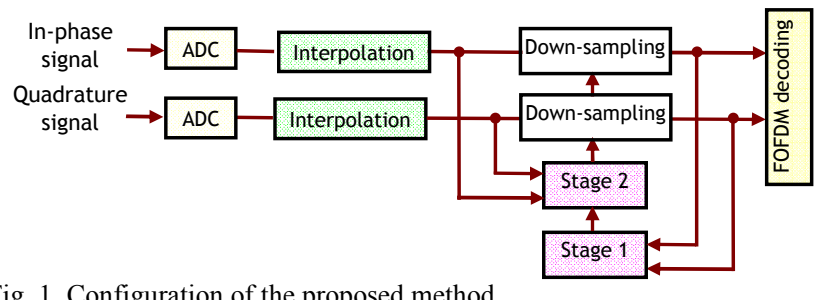

Fig. 1. Configuration of the proposed method.

Fig. 1 illustrates the configuration of the proposed method, in which the received samples from the analogue-to-digital converter (ADC) are interpolated and down-sampled to the sampling rate of the original transmitted FOFDM signal. The proposed method comprises two stages to not only identify the SOF symbol but also determine the correct timing position for the DCT window. The ADC in Fig. 1 is not necessarily over-sampled, and the interpolation is required to increase the resolution for precise timing recovery in FOFDM. Additional study shows that the impact of using different interpolation methods on the proposed method is small.

The first stage is based on the concept outlined in [5], with appropriate modification for FOFDM. The time-domain signal of the SOF symbol is designed to be periodic, e.g. $s(n)=s(n+N / 2)$, where $s(n)$ is the transmitted signal and $N$ is the DCT point size. To achieve this, the values of sub-carriers are 
set to be only non-zero and real for $a_{\mathrm{k}}$ with $k=4 p$ (Fig. 2), where $p$ is an integer, because the sub-channel spacing in FOFDM is only half of the symbol rate per sub-carrier. Consequently, the timing metric at stage one, $M_{\mathrm{s}}$, as defined in [5,7], can yield a peak for the SOF symbol. However, there is a plateau in this metric, which introduces ambiguity at the symbol boundary.
Sub-carrier data before IDCT

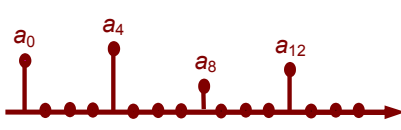

(a)
Time domain signal after IDCT

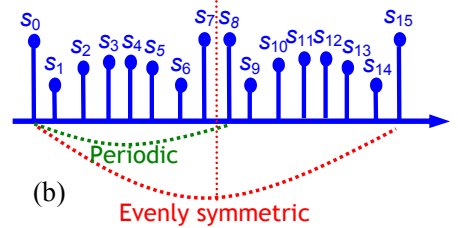

Fig.2. (a) Sub-carrier allocation and (b) the time-domain signal of the SOF symbol in optical FOFDM. The sub-carrier number $N$ is assumed to be 16 .

To overcome this ambiguity, the second stage is used. From Fig. 2, it can be seen that the time-domain signal of the SOF symbol after IDCT is not only periodic but also evenly symmetric, e.g. $s(n)=s(N-1-n)$. Mathematically, any received $N$-point in-phase (quadrature) signal $r_{\mathrm{I}}(n)\left(r_{\mathrm{Q}}(n)\right)$ can be expressed as the sum of an even function $r_{\mathrm{I} \text {,even }}(n)\left(r_{\mathrm{Q} \text {,even }}(n)\right)$ and an odd function $r_{\mathrm{I}, \text { odd }}(n)\left(r_{\mathrm{Q} \text {,odd }}(n)\right)$. We propose $M_{\mathrm{s} 2}$ at stage two:

$$
M_{s 2}=\frac{\sum_{n=0}^{N / 2-1}\left|r_{I, \text { odd }}\left(n+m_{\text {error }}\right)+j \cdot r_{Q, \text { odd }}\left(n+m_{\text {error }}\right)\right|^{2}}{\sum_{n=0}^{N / 2-1}\left|r_{I, \text { even }}\left(n+m_{\text {error }}\right)+j \cdot r_{Q, \text { even }}\left(n+m_{\text {error }}\right)\right|^{2}}
$$

where $m_{\text {error }}$ is the sample index representing the timing error. Ideally, due to the even symmetry of the time-domain signal for the SOF symbol, $M_{\mathrm{s} 2}$ is zero for $m_{\text {error }}=0$. In practice, the minimum of $M_{\mathrm{s} 2}$ is found to determine the symbol boundary. Note that in conventional OFDM, Hermitian symmetric property of DFT was used for timing estimation [7]. However, Hermitian symmetry cannot be preserved in the presence of CD, which will be shown in Fig. 6(a). In addition, the real output of DCT enables the proposed method resilient to the in-phase/ quadrature imbalance problem at the receiver. Thirdly, the guard interval (GI) in this paper is designed to be cyclic prefix and suffix, but (1) is also applied to a system with other designs of GI, e.g. zero padding [2] or symmetrical extension [3]. Finally, Schmidl's frequency-offset estimation algorithm is also applicable, and FOFDM is more robust to the residual frequency offset than conventional OFDM [2].

\section{EXPERIMENTAL SETUP AND RESULTS}

Fig. 3 shows the experimental setup. The binary phase shifted keying (BPSK) based FOFDM signal was encoded using Matlab. The inverse-DCT (IDCT) and DCT used 256 points, of which 180 sub-carriers (sub-carriers \#2-\#181) were used for data transmission. The first sub-carrier was not modulated, allowing for AC-coupled driving amplifiers and receivers. After IDCT and parallel-to-serial (P/S) conversion, 24 samples (1ns) were added to each symbol as a guard interval. The generated FOFDM signal was downloaded to an arbitrary waveform generator (AWG) with $24 \mathrm{GS} / \mathrm{s}$ and a resolution of 8 bits. The nominal signal line rate was $16.88 \mathrm{Gbit} / \mathrm{s}(24 \times 180 / 256)$. A fiber laser with $6 \mathrm{kHz}$ linewidth was used to generate the optical carrier. A Mach-Zehnder modulator (MZM) was used for signal modulation. The modulated optical signal was then transmitted over a $124 \mathrm{~km}$ field-installed single-mode fiber (SMF) operated by BT Ireland between Cork City and Clonakilty with $-4 \mathrm{dBm}$ launch power. At the receiver, a variable optical attenuator (VOA) was used to vary the input power to the erbium doped fiber amplifier (EDFA). The pre-amplifier was followed by an optical band pass filter (OBPF) with a $3 \mathrm{~dB}$ bandwidth of $0.3 \mathrm{~nm}$, a second EDFA, and another optical filter with a $3 \mathrm{~dB}$ bandwidth of $1 \mathrm{~nm}$. A polarization controller (PC) was used to align the polarization of the filtered FOFDM signal before entering the signal path of a $90^{\circ}$ hybrid. A tap of the transmitter laser signal was used as the local oscillator at the receiver. The optical outputs of the hybrid were connected to two balanced photodiodes with $40 \mathrm{GHz} 3 \mathrm{~dB}$ bandwidths, amplified by $40 \mathrm{GHz}$ electrical amplifiers, and captured using a $50 \mathrm{GS} / \mathrm{s}$ real-time oscilloscope. The decoding algorithms included the symbol synchronization technique as proposed in this paper, dispersion compensation, IDCT, and phase estimation. Because the diagonalizing property of DFT could not be generally applied to DCT, CD compensation in this paper was performed before DCT. Simple and effective CD compensation was an important issue in FOFDM, and in wireless, schemes such as minimum mean-square-error detection [2] or pre-filter [3], were proposed. Seventy (70) FOFDM frames were measured, where each frame consisted of eighty (80) FOFDM symbols and one SOF symbol. The total number of measured bits was $180 \times 80 \times 70=1,008,000$.

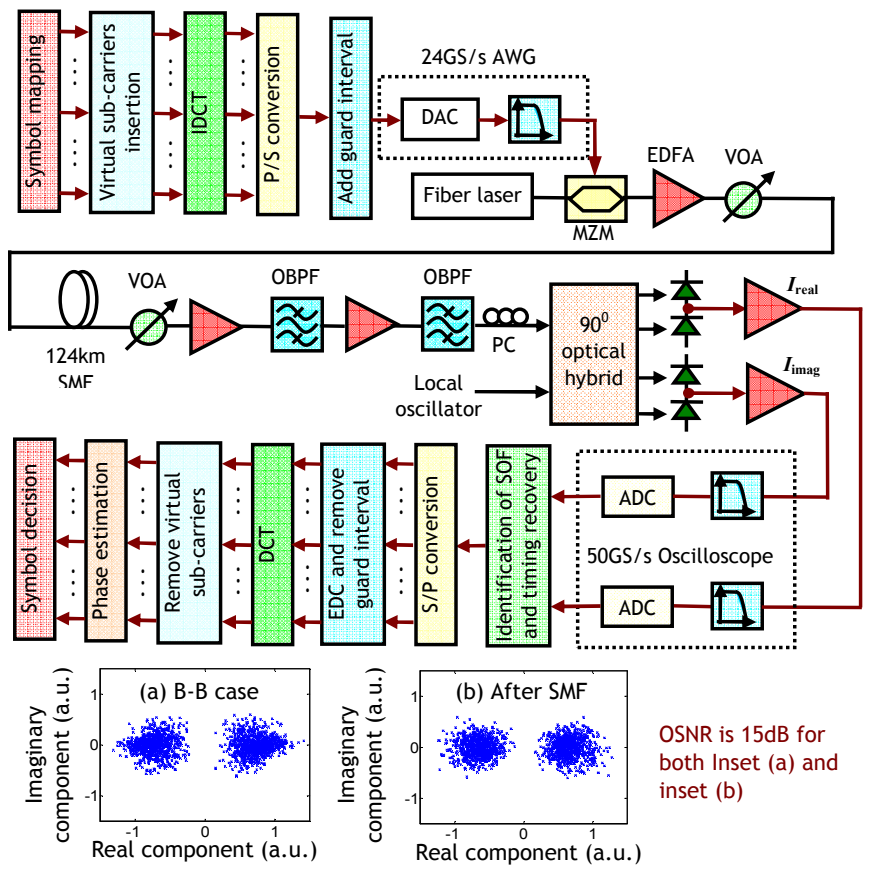

Fig. 3. Experimental setup for BPSK-based FOFDM. Insets (a) and (b) are the recovered constellation diagrams of FOFDM signal for (a) the back-to-back case; (b) after $124 \mathrm{~km}$ field-installed SMF transmission at $15 \mathrm{~dB}$ OSNR.

Fig. 4(a) and (b) depict the timing metric generated at the first stage versus the timing error. The peak that indicated the SOF can be clearly seen. However, the metric reached a plateau, with an ambiguity of $\sim 2$ ns. This uncertainty was overcome by using the proposed metric (Fig. 4(c)\&(d)). The figures show that a dip 
was generated at the optimal symbol boundary at an OSNR of $5.5 \mathrm{~dB}$ for both the back-to-back case and the case after $124 \mathrm{~km}$ $\mathrm{SMF}$. At the minimum point, the value of $M_{\mathrm{S} 2}$ was close to zero.
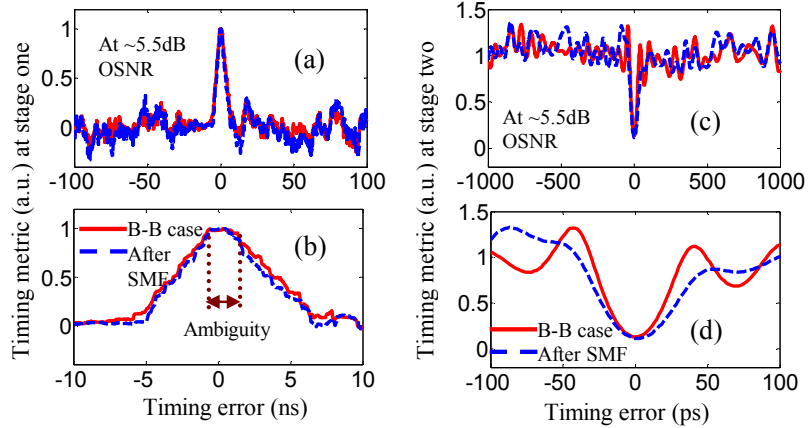

Fig. 4. Example of (a)\&(b) the metric generated at stage one and (c)\&(d) the metric generated at stage two versus the timing error. In all figures, solid and dashed lines represent the back-to-back case and the case after $124 \mathrm{~km}$ field-installed SMF transmission. The OSNR value was around 5.5dB.
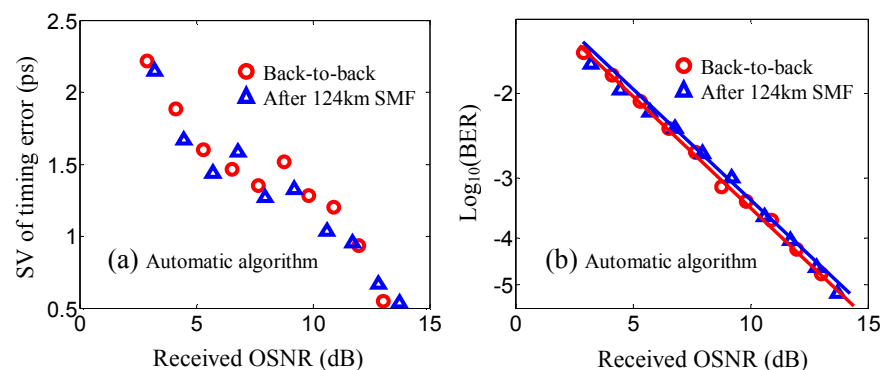

Fig. 5. (a) The SV of the timing error and (b) $\log _{10}(\mathrm{BER})$ versus the received OSNR (dB) for the back-to-back case (circles) and the case after 124km SMF (triangles) by using the proposed method.
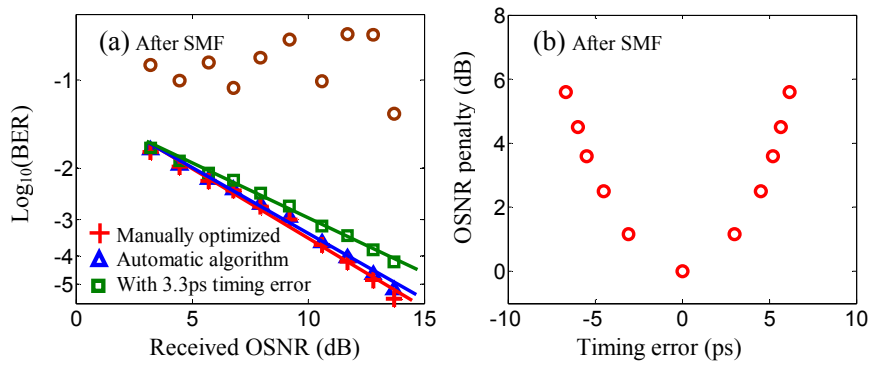

Fig. 6. (a) $\log _{10}(\mathrm{BER})$ versus the received OSNR (dB) using optimized sampling phase (pluses), using the proposed method (triangles), with $3.3 \mathrm{ps}$ timing error (squares), and using Park's metric [7] (circles); (b) OSNR penalty $(\mathrm{dB})$ versus the timing error at BER of $10^{-3}$ after $124 \mathrm{~km} \mathrm{SMF}$.

We define the standard variance (SV) of the timing error:

$$
S V \text { of timing error }=\left(\sum_{p=1}^{J}\left(t_{p, \text { search }}-t_{p, \text { optimal }}\right)^{2} / J\right)^{1 / 2}
$$

where $t_{\mathrm{p}, \text { search }}$ and $t_{\mathrm{p} \text {,optimal }}$ are the timing value obtained by using the proposed automatic algorithm and the manually optimized timing value for the $p^{\text {th }}$ frame respectively. $J$ is the number of FOFDM frame (70 in this paper). Fig. 5(a) shows the calculated $\mathrm{SV}$ of the timing error versus the received OSNR. It can be clearly seen that SV of the timing error was less than $1 \mathrm{ps}$ for OSNR values larger than $12 \mathrm{~dB}$, and, in a general trend, increased with the received OSNR. However, even when the OSNR was reduced to $3 \mathrm{~dB}$, the SV of the timing error was still less than 2.2ps. By using this technique for automatic symbol synchronization, we measured the bit error rate (BER) of the 16.88Gbit/s FOFDM system, with the results illustrated in Fig. 5(b). The figure shows that a negligible penalty was observed after $124 \mathrm{~km}$ field-installed SMF transmission and the required OSNR at BER of $1 \times 10^{-3}$ was $\sim 9 \mathrm{~dB}$ for both the back-to-back case and the case after transmission of the $124 \mathrm{~km}$ field-installed SMF. Fig. 6(a) compares the performance using optimized sampling phase (pluses), using the proposed method (triangles), with 3.3ps timing error (squares), and using Park's metric [7] (circles) after $124 \mathrm{~km} \mathrm{SMF}$. The figure confirms that the penalty from the residual timing error by using the proposed algorithm was negligible, whilst Park's metric could not be applied to the FOFDM system in the presence of CD. The maximum CD tolerance of the proposed method depended on the length of the GI. The impact of the timing error varied with the BER value, and the OSNR penalty induced by 3.3ps timing error was around $1 \mathrm{~dB}$ at BER of $10^{-3}$. More investigation on the influence of the timing error is depicted in Fig. 6(b), where it is shown that the tolerance at $3 \mathrm{~dB}$ penalty was around $\pm 5 \mathrm{ps}$.

\section{CONCLUSIONS}

We have proposed a new and simple automatic algorithm that exploits the evenly symmetric property of the DCT to achieve precise symbol synchronization in optical FOFDM, and by using this method, have demonstrated $16.88 \mathrm{Gbit} / \mathrm{s}$ FOFDM transmission over $124 \mathrm{~km}$ field-installed SMF. The results show that the proposed method has a SV of the timing error less than $2.2 \mathrm{ps}$ and $1 \mathrm{ps}$ for $3 \mathrm{~dB}$ and $12 \mathrm{~dB}$ OSNR respectively, and is resilient to the $\mathrm{CD}$ without any need for modification of the algorithm. We thank W. McAuliffe and D. Cassidy from BT Ireland for provision of the installed fiber, and $\mathrm{X}$. Liu from Alcatel-Lucent for fruitful discussions.

\section{REFERENCES}

[1]. J. Zhao, A.D. Ellis, "A novel optical fast OFDM with reduced channel spacing equal to half of the symbol rate per carrier," Optical Fiber Communication Conference (2010), paper OMR1.

[2]. P. Tan and N.C. Beaulieu, "A comparison of DCT-based OFDM and DFT-based OFDM in frequency offset and fading channels," IEEE Trans. Commun., vol. 54, pp. 2113-2125, 2006.

[3]. N.A. Dhahir, H. Minn, and S. Satish, "Optimum DCT-based multicarrier transceivers for frequency-selective channels," IEEE Trans. Commun.. vol. 54, pp. 911-921, 2006.

[4]. J. Zhao, S.K. Ibrahim, D. Rafique, P. Gunning, A.D. Ellis, "A novel method for precise symbol synchronization in double-side band optical fast OFDM," to appear in Optical Fiber Communication Conference (2011).

[5]. T.M. Schmidl and D.C. Cox, "Robust frequency and timing synchronization for OFDM," IEEE Trans. Commun., vol. 45, pp. 1613-1621, 1997.

[6]. C.J. Youn, X. Liu, S. Chandrasekhar, Y.H. Kwon, J.H. Kim, J.S. Choe, K. S. Choi, and E.S. Nam, "An efficient and frequency-offset tolerant channel estimation and synchronization method for PDM CO-OFDM transmission," European Conference on Optical Communication (2010), paper P.4.06.

[7]. B. Park, H. Cheon, C. Kang, and D. Hong, "A novel timing estimation method for OFDM systems," IEEE Commun. Lett., vol. 7, pp. 239, 2003.

[8]. X.Q. Jin, R.P. Giddings, E.H. Salas, and J.M. Tang, "First experimental demonstration of end-to-end real-time optical OFDM symbol synchronization using subtraction and Gaussian windowing in $25 \mathrm{~km}$ SMF IMDD systems," European Conference on Optical Communication (2010), paper P.4.19. 